\title{
Primary Malignant Melanoma of the Lumbar Spine
}

\author{
Mathew CJ ${ }^{1}$, Karim F ${ }^{1}$, Ali $\mathbf{M}^{2 *}$, Katsigiorgis $\mathbf{G}^{\mathbf{1}}$, \\ Lerman $V^{1}$ and Avanesov $K^{1}$ \\ ${ }^{1}$ Department of Orthopaedic Surgery, Northwell \\ Plainview Hospital, USA \\ ${ }^{2}$ New York Institute of Technology College of Osteopathic \\ Medicine, Old Westbury, USA \\ *Correspondling author: Muzaffar Ali, New York \\ Institute of Technology College of Osteopathic Medicine, \\ Northern Boulevard, Old Westbury, USA
}

Received: J anuary 20, 2017; Accepted: February 13, 2017; Published: February 15, 2017

\begin{abstract}
Low Back Pain (LBP) or Lumbago is a leading cause of disability in the United States. There are several etiologies including but not limited to muscle strains, discherniation, vertebral instability and fractures. Most serious however are metastatic neoplastic processes, which invade the spine, spinal cord, and nerve roots potentially causing significant neurologic and functional compromise. Primary tumors of the spine are extremely rare, even rarer are primary melanomas of the spine [1]. We present a rare case of a 55-year-old male that was admitted with unrelenting back pain and inability to ambulate. Imaging shows pathologic L3 vertebral body compression fracture, biopsy confirmed malignant melanoma. Clinical examinations from dermatology, gastroenterology, ophthalmology, and oncology confirmed that there were no primary sources of the malignancy identified resulting in the spinal lesion being the only source and location of the tumor [1]. The patient's radicular symptoms and function improved post-operatively, patient was informed that data of his case will be submitted for publication. However, he later succumbed to a myocardial infarction two years later.
\end{abstract}

Keywords: Low back pain; Malignant melanoma; Computed tomography

\section{Introduction}

Low back pain (LBP) is a leading cause of disability in the United States. LBP and its neurologic sequelae account for countless visits to emergency rooms and physician offices. Back pain can be as simple as a paraspinal muscle strain but can also be more complex, involving fractures and slippage of the vertebrae as well as herniated discs causing nerve root compression. Due to the common nature of back pain, it is often managed conservatively with physical therapy, antiinflammatory, anti-spasmodic and other analgesics. We present a unique case of LBP resulting from a malignant melanoma in the rare isolated site of the vertebral body.

Our patient was a middle-aged Caucasian male who presented to the emergency room with atraumatic severe low back pain and bilateral lower extremity weakness. Pathologic fracture of L3 vertebral body was noted and upon further work-up, a unique etiology of an isolated malignant melanoma was found within the vertebral body. After tumor resection and stabilization, the patient's pain and mobility improved. We present this case to increase the awareness of atypical causes of LBP and to highlight the unique presentation of an isolated malignant melanoma arising within the vertebral body [2].

\section{Case Presentation}

A55-year-oldcommunity ambulating male with a significant past medical history of alcohol abuse, psoriasis and hypertension presented to the emergency room with a 3-day history of atraumatic low back pain and decreasing ability to ambulate. He denied any fevers or chills, recent weight loss or other constitutional symptoms. He denied any saddle paresthesias or loss of bowel or bladder control [2]. Due to his uncontrolled psoriasis, the patient frequently went to tanning salonsto control his outbreaks. On physical exam, he demonstrated no gross deformities, lesions or sores. He did present with lumbar paraspinal tenderness and spasm, with lower extremity weakness secondary to pain on bilateral hip flexion. He was intact to sensation to light touch in his bilateral lower extremities, with normal patellar, Achilles, Babinski and rectal tone reflexes and normal dorsalis pedis and posterior tibialis pulses. Upon presentation to the emergency department, radiographs of the lumbosacral spine revealed bony destruction at the endplates L3 with chronic deformation of the anterior aspect of the vertebral body (Figures 1).

Initial clinical presentation and radiographic images warranted additional clinical studies and further imaging was obtained. Computed Tomography (CT) demonstrated a heterogeneously enhancing lytic expansile lesion from the vertebral body extending to the soft tissue suggestive of an oncologic process (Figures 2).

Multiplanar sequence Magnetic Resonance Imaging (MRI) was obtained (Figures 3) to assess the extent of the tumor mass and involvement of neurological structures, which revealed extensive signal enhancement of the L3 vertebral body and surrounding

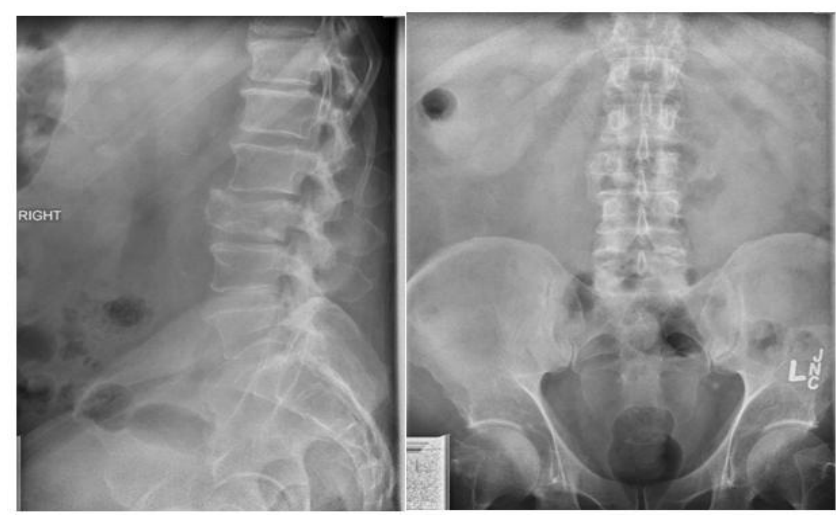

Figures 1: AP and Lateral Radiographs demonstrating compression fracture of L3.
Austin J Orthopade \& Rheumatol - Volume 4 Issue 1 - 2017 ISSN: 2472-369X | www.austinpublishing group.com

Ali et al. (C) All rights are reserved
Citation: Mathew CJ, Karim F, Ali M, Katsigiorgis G, Lerman V and Avanesov K. Primary Malignant Melanoma of the Lumbar Spine. Austin J Orthopade \& Rheumatol. 2017; 4(1): 1047. 


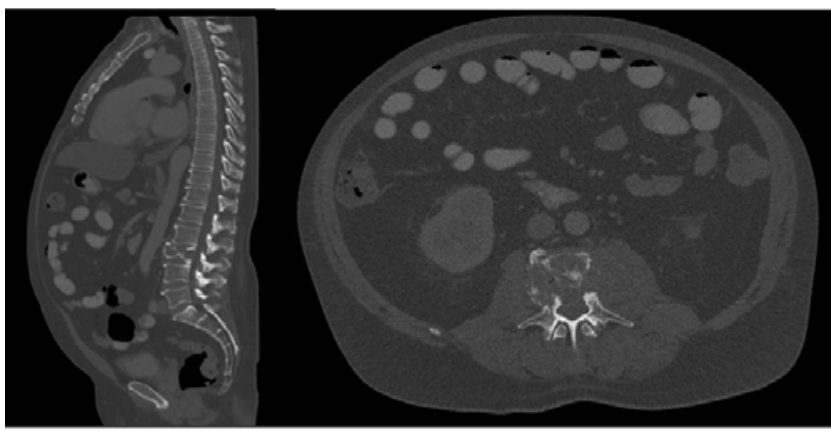

Figures 2: Computed Tomography (CT) of thoracic, and lumbosacral spine. Mid Sagittal (a) and Axial image (b) at L3 demonstrating heterogeneous expansile lesion at L3.

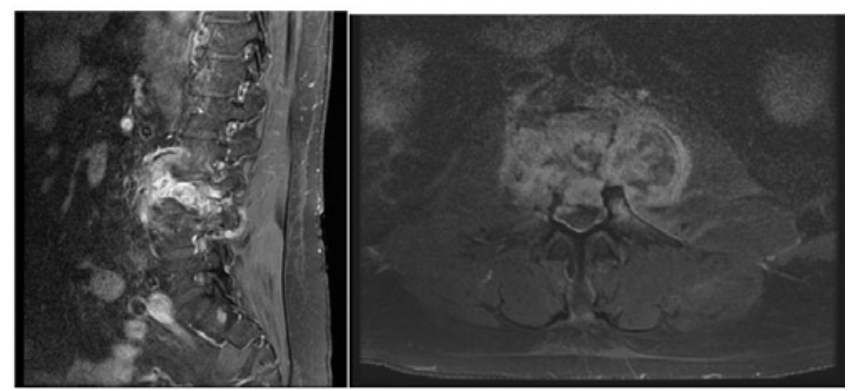

Figures 3: MRI of Lumbosacral Midsagittal T2 (a) and Axial T1(b) at L3 demonstrating enhancing lesion of L3 with extension into surrounding soft tissue structures.

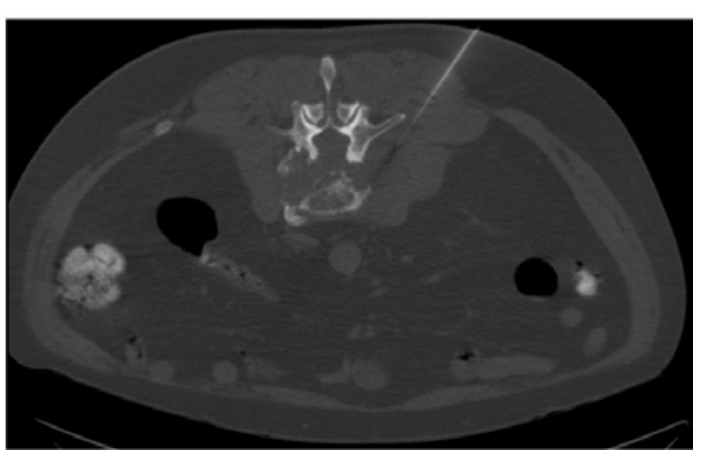

Figure 4: Axial Cut of CT at L3 demonstrating needle placement into the pathologic lesion.

structures. In addition to the loss of bony architecture there were several areas of necrosis demonstrated by signal enhancement of paraspinal musculature. Further imaging was suggestive of a metastatic or primary spinal lesion.

Interventional radiology was consulted to obtain a biopsy of the vertebral neoplastic process. CT guided biopsy of the lesion (Figure 4) was performed and a specimen from the left lateral side of vertebral body was sent for evaluation to pathology in formalin. Gross examination of the tissue consisted of two cores of dark brownish to black tan tissue measuring $(0.6 \times 0.1 \times 0.1 \mathrm{~cm})$ and $(1.5$ x $0.1 \times 0.1 \mathrm{~cm})$. Both cores demonstrated extensive necrosis. Fine needle aspirate showed individual cells and small clusters with blue granular cytoplasm, nuclei with euchromatic chromatin and rare

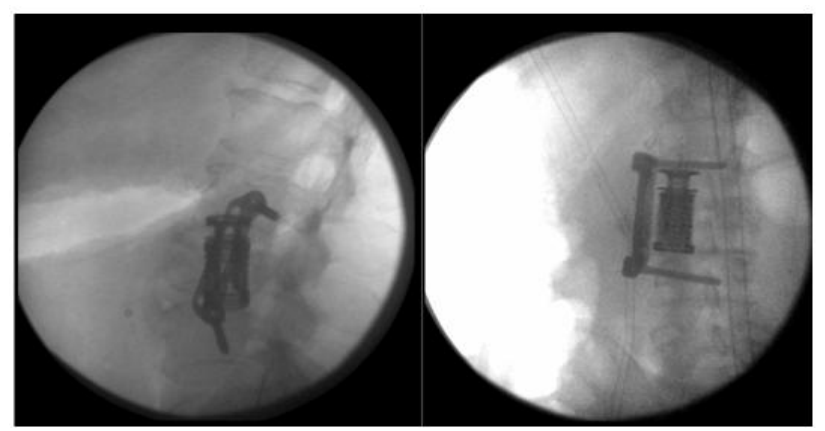

Figures 5: Intraoperative AP and Lateral Lumbar Spine fluoroscopic imaging demonstrating L3 Corpectomy and cage placement.

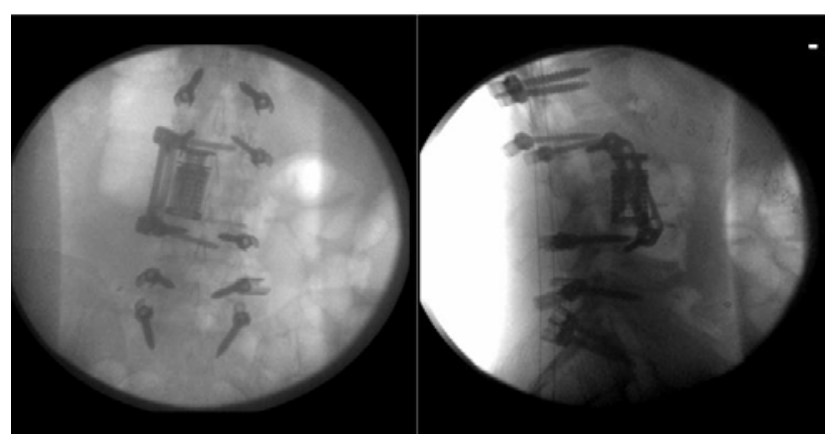

Figures 6: Intraoperative AP and Lateral Spine fluoroscopic imaging after posterior stabilization.

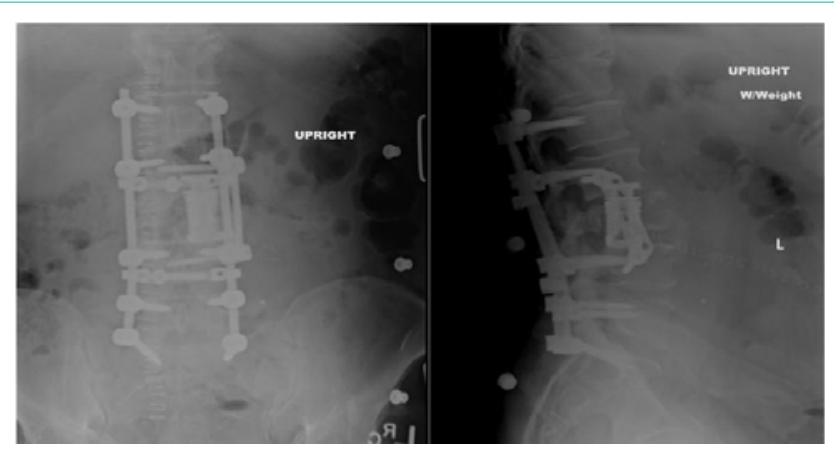

Figures 7: Postoperative AP and Lateral Lumbar Spine radiographs demonstrating L3 corpectomy, expandable cage placement and posterior instrumentation.

intranuclear inclusions. Immunohistochemistry test results were positive for Human Melanoma Black (HMB) 45 in 70\%, S100 in 90\%, and Tyrosinase in $100 \%$ of the confirming the diagnosis of malignant melanoma [3].

Due to the likelihood that the vertebral melanoma was of metastatic nature from potentially gastroenterologic, dermatologic, opthomalogic sources, consultations from these specialties were called and showed no other sources of the malignancy. Further imaging and work up demonstrated no other lesions [4]. After determination that the diagnosis was likely a primary melanoma and the only site of involvement, surgical intervention was warranted to halt the spread of the malignancy, decrease tumor load, alleviate neurological symptoms and improve function. 
Surgical stabilization and decompression was planned after a debulking of the tumor with specific attention paid to restoring the spinal column alignment to prevent more neural compromise and alleviate pressure on the nerves. Due to character of the tumor and its intimate location to vital fragile structures, a lateral debulking was pursued versus wide resection followed by a staged posterior fusion with instrumentation to enhance the rigidity for the fusion mass.

The patient was taken to the operating room and a lateral approach was utilized for tumor resection, debulking and corpectomy was performed at L3. An expandable cage with allograft was placed with additional plate stabilization (Figures 5) [5]. Several intraoperative pathology samples were taken which later confirmed the diagnosis of malignant melanoma.

Due to the invasiveness and surgical demand of the procedure, a decision to stage the intervention was made and a L1-S1 instrumented posterior spinal fusion was later performed without complication. Intraoperative fluoroscopic images were obtained to confirm placement of the cage and screws (Figures 6, Figure 7)

The patient's postoperative course was benign and a multidisciplinary approach to his care was continued. He was discharged to rehabilitation in stable condition and was later treated with stereotactic radiation. Despite continuing to have mild residual back pain his radicular leg pain improved. Upon multidisciplinary follow up, no new neoplastic processes were observed and the hardware remained intact and the incision healed well. Patient was informed that his case will be submitted for publication because of the rare nature of this cancer. Unfortunately, the patient later succumbed to a myocardial infarction two years later.

\section{Discussion}

Our patient having a relatively benign presentation to the emergency room demonstrates the importance of an appropriate work-up for the common problem of low back pain. The patient had not been experiencing any weight loss, fevers, chills or other constitutional symptoms nor demonstrated any abnormal lab values that could have easily left the impression of osseous, muscular, neurologic discogenic etiologies. Understanding that an atraumatic vertebral compression fracture on radiographs in an otherwise healthy individual is cause for alarm for metabolic, infectious or neoplastic processes, thus requiring more advanced imaging. Imaging was crucial to starting the diagnosis due to the atypical nature of the fracture and the localized soft tissue involvement seen on radiographs, CT and MRI.

Oncological involvement of the spine is often secondary to metastasis. Osseous tumors are often secondary to due to metastatic disease from neoplasms of the breast, lung, thyroid, kidneys and prostate, however there are few lesions that are primary to the spine. In the case of metastatic melanoma of the spine it is usually indicative of a highly aggressive pathology; however primary melanoma tends to remain in the spine for a relatively extended period of time. Studies have shown that although cutaneous metastatic melanoma has a life expectancy of 6 months, patients with primary melanoma of the CNS can live up to 7 years after initial diagnosis [6].

Primary melanoma of the central nervous system is rare and accounts for approximately $1 \%$ of all cases of melanoma [7]. Primary melanoma of the spine is likely contained due melanocytes in the leptomeninges, which can easily spread into the local bone in a necrotic and destructive fashion. The initial research conducted by Virchow in the mid 1800s show that the melanomas developed from precursor cells in the leptomeninges known as melanoblasts [8]. These melanoblasts can differentiate into a variety of masses included melanocytomas, pigmented schwannomas and pigmented neuroblastomas. As with all tumors obtaining a biopsy is vital in order to secure the diagnosis. Metastasis of primary melanoma is extremely rare due the lack of lymphatic spread from fewer lymphatic vessels within neural tissues [8].

There have been fewer than 50 cases previously reported of spinal melanomas since first described by Hirschberg in 1906 [9]. Most cases of spinal melanomas are metastatic, with any melanoma to the bone being a poor prognostic indicator [4]. Mortality after metastasis is elevated and often indicative of an aggressive tumor. Extramedullary melanomas of the spine are also extremely rare often arising from nerve roots, causing extensive destruction [10]. In these situations the patient is often burdened with other regions of tumor; however, our patient presented with a unique case of an isolated malignant melanoma of the L3 vertebra and surrounding soft tissue without primary source.

Despite thorough clinical evaluation by dermatologists, ophthalmologists and gastroenterologists it was determined that the malignancy was primary in nature because diagnosis requires exclusion of a primary cutaneous, gastrointestinal or ocular lesion [5]. CT guided biopsy and Immunohistological analysis was crucial to defining the lesion and it's management. Malignant melanoma typically presents with individual cells of small clusters with blue granular cytoplasm, nuclei with euchromatic chromatin and rare intranuclear inclusions. Immunohistochemistry test are typically positive for Human Melanoma Black (HMB) 45, S100 and Tyrosinase as in our patient [3].

It was discussed whether the patient's history of frequent use of tanning beds may have induced a cutaneous melanoma that spread to his spine, however it was thought that this would produce other areas of metastasis rather than an isolated region. This was further confirmed by the fact that he met progression and life expectancy goals of an individual who had a primary CNS tumor and was not lost to follow up after stereotactic radiation therapy.

The majority of cases of spinal melanoma are indicative of late stage disease and spinal metastasis. In such circumstances, spinal involvement is generally treated through palliative measures or radical en bloc resection. Uniquely this patient's disease appeared to be isolated to the lumbar spine, as most cases are often in the thoracic spine. Important to also note is that in cases of metastasis patients, patient can experience constitutional symptoms like night sweats, fevers, and chills, unintentional weight loss, however primary spinal tumors may not have this classic presentation. The patient's primary symptoms were neurologic symptoms of low back pain and bilateral lower extremity weakness and pain likely from close relationship of the psoas to the malignancy.

Current systemic treatment strategies for melanoma continue 
to be poor. The disease process has continued to be unphased by a single agent or combination chemotherapy regimens. These regimens have not been effective at improving overall survival. Even novel bio therapeutics such as antisense bcl-2 oligonucleotide oblimersin, which is a reactive -oxide species inhibitor has not played a role in increasing survival [11]. With the advent of custom immunobiologics and targeted antibodies there appears to be some ground being gained on understanding the complex disease process.

In the case of primary spinal melanoma, we must continue to recognize that a problem as low back pain can have multiple etiologies ranging from degenerative, traumatic, infections and neoplastic. Without a thorough history, physical exam and adequate imaging, the true diagnosis of low back pain can be overlooked and unrecognized. Due to the common nature of LBP, it is imperative as a physician to be knowledgeable and cognizant of red flags like constitutional symptoms that are clues to atypical causes of low back pain.

\section{Conclusion}

The etiology of back pain can be both simple and complex. There is no substitute for an adequate history, physical exam, and imaging in understanding of any musculoskeletal disease process. Primary spinal melanomas are rare tumors, but due to their capability to generally be isolated to the CNS have a much better prognosis than metastatic melanoma provided there is adequate patient follow up. It is important that providers look to the clinical clues and understand in a younger patient a vertebral compression fracture without history of trauma may be indicative of a much more serious disease process. As with all oncological conditions early detection and treatment continue to be the mainstays of improved treatment and survival.

\section{References}

1. Gokaslan ZL, Aladag MA, Ellerhorst JA. Melanoma metastatic to the spine: a review of 133 cases. Melanoma Res. 2000; 10: 78-80.

2. Donaldson WF, Peppelman WC, Yaw KM. Symptomatic metastatic malignant melanoma to the spine. J Spinal Disord. 1993; 6: 360-363.

3. Fuld AD, Speck ME, Harris BT, Simmons NE, Corless CL, Tsongalis GJ, et al Primary melanoma of the spinal cord: a case report, molecular footprint, and review of the literature. J Clin Oncol. 2011; 29: e499-502.

4. Spiegel DA, Sampson JH, Richardson WJ, Friedman AH, Rossitch E, Hardaker WT Jr, et al. Metastatic melanoma to the spine. Demographics, risk factors, and prognosis in 114 patients. Spine. 1995; 20: 2141-2146.

5. Dominkus M, Krepler P, Schwameis E, Kotz R. [Surgical therapy of spinal metastases]. Orthopade. 1998; 27: 282-286.

6. Larson TC, Houser OW, Onofrio BM, Peipgras DG. Primary spinal melanoma. J Neurosurg. 1987; 66: 47-49.

7. Kounin GK, Romansky KV, Traykov LD, Shotekov PM, Stoilova DZ. Primary spinal melanoma with bilateral papilledema. ClinNeurolNeurosurg. 2005; 107: 525-527.

8. Katalinic D, Anic B, Stern-Padovan R, Mayer M, Sentic M, Cikes N, et al Low back pain as the presenting sign in a patient with primary extradural melanoma of the thoracic spine--a metastatic disease 17 years after complete surgical resection. World J SurgOncol. 2011; 9: 150.

9. Brat DJ, Giannini C, Scheithauer BW, Burger PC. Primary melanocytic neoplasms of the central nervous system. Am J Surg Pathol. 1999; 23: 745754.

10. Naing A, Messina JL, Vrionis FR, Daud Al. Uncommon manifestations of common malignancies: case 3. Malignant melanoma arising from a spinal nerve root. J Clin Oncol. 2004; 22: 3194-3195.

11. O'day SJ, Atkins MB, Boasberg P, et al. Phase II multicenter trial of maintenance biotherapy after induction concurrent Biochemotherapy for patients with metastatic melanoma. J ClinOncol. 2009; 27: 6207-6212.
Austin J Orthopade \& Rheumatol - Volume 4 Issue 1 - 2017 ISSN: 2472-369X | www.austinpublishing group.com Ali et al. (C) All rights are reserved
Citation: Mathew CJ, Karim F, Ali M, Katsigiorgis G, Lerman V and Avanesov K. Primary Malignant Melanoma of the Lumbar Spine. Austin J Orthopade \& Rheumatol. 2017; 4(1): 1047. 